\title{
Comprehensive Geriatric Assessment Using the Yoitoko Check-Up, a Novel Health Check-Up Providing Positive Feedback to Older Adults: A Before-After Study
}

\author{
Shinsuke Yahata (D) \\ Minoru Tamura ${ }^{2}$ \\ Atsushi Yamaoka ${ }^{2}$ \\ Yoshihide Fujioka ${ }^{2}$ \\ Masanobu Okayama' \\ 'Division of Community Medicine and \\ Medical Education, Kobe University \\ Graduate School of Medicine, Kobe, \\ Hyogo, Japan; ${ }^{2}$ Kobe University Graduate \\ School of Economics, Kobe, Hyogo, Japan
}

Correspondence: Masanobu Okayama Division of Community Medicine and Medical Education, Kobe University Graduate School of Medicine, 2-I-5, Arata-Cho, Hyogo-Ku, Kobe, Hyogo, 6520032, Japan

Tel $+81-78-382-6732$

Fax +8I-78-382-6283

Email okayamam@med.kobe-u.ac.jp
Purpose: The Yoitoko check-up, a novel health check-up providing positive feedback, has been developed to promote health among older adults, and consists of several comprehensive geriatric assessment items. This report aimed to describe the details of the Yoitoko check-up and to explore the future possibility of the check-up by evaluating the participants' shortterm behavioral changes in terms of comprehensive functioning, using a before-after study design.

Patients and Methods: Four Yoitoko check-ups were conducted, at 3-month intervals, between December 2018 and September 2019. Study participants aged $\geq 65$ years included those who had undergone $\geq 2$ Yoitoko check-ups. The results of each visit after the second check-ups were retrospectively compared with those of the baseline, and the mean changes and the odds ratios were calculated using a paired $t$-test or a McNemar test, respectively.

Results: Of 84 participants, the results of $16(19.0 \%)$ participants were analyzed. The mean (standard deviation) age was 75.3 (4.7) years. The mean Tokyo Metropolitan Institute of Gerontology Index of Competence score, a measure of high-level functional capacity, increased 0.9 (95\% confidence interval; range, $0.2-1.5)$ points between the first and second visits.

Conclusion: We developed the Yoitoko check-up and introduced the details of it. Our study findings suggested that the Yoitoko check-up may further motivate older adults to improve their health and promote positive behavioral changes. Future studies are needed to evaluate the effectiveness of this novel assessment method.

Keywords: health promotion, preventive care, behavioral change, motivation, high-level functional capacity

\section{Introduction}

The global population is aging ${ }^{1,2}$ and Japan, in particular, has the most aged population in the world. ${ }^{2}$ Older adults are reportedly more prone to multiple comorbidities and demand a large amount of health care. ${ }^{3}$ Societal influences due to an aging population would have a significant effect on building a sustainable society. Overcoming the challenges posed by an aging population requires the accomplishment of Sustainable Development Goals (SDGs) such as promoting health and preventive care, reducing age- and sex-related barriers to employment, and establishing social security systems. ${ }^{2}$ SDGs require older people to stay 
healthier, work longer, save for retirement, and be provided with adequate benefits against poverty and inequality. ${ }^{2}$ In 2013, the Japanese government developed the second term of the National Health Promotion Movement in the $21^{\text {st }}$ century (Health Japan 21 [the second term]) and has been implementing health promotion action plans with the goal of extending healthy life expectancy and reducing health disparities. ${ }^{4}$

To foster improvement in the health of older adults, local governments in Japan have been conducting health checks for older adults aged $\geq 75$ years. These health checkups have been constructed with reference to health checkups for Japanese citizens aged 40-74 years, focusing on lifestyle diseases such as diabetes. ${ }^{5}$ Although this intervention works for younger adults, ${ }^{6,7}$ there is no evidence of its effectiveness for older adults. Conventional health checkups identify health issues, so-called "bad points", with a view to encouraging older adults to resolve any issues identified during a check-up. In 2016, the uptake rate was reported to be $28.0 \% .{ }^{8}$ In a survey that investigated reasons why older adults had not undertaken a health check-up or a comprehensive medical examination, $54.2 \%$ of those aged $\geq 75$ years responded: "I can receive a check-up at a medical institution whenever a health concern arises", which was higher than responses given by those aged $>20$ years $(33.5 \% \text {. })^{9}$ As people age, it may be that they are less motivated to prevent illness and improve their health, and it is possible that they consider it better to manage health issues as they emerge. A conventional health check-up aiming to identify health issues and encourage improvement appears unlikely to motivate people to promote their health.

To address the shortcomings in the conventional health check-up, we developed a novel style of health check-up, namely the Yoitoko check-up. In Japanese, the word "Yoitoko" means "good point." Items included in the Yoitoko check-up are based on a comprehensive geriatric assessment (CGA) for older adults. The CGA is defined as

a multidimensional, interdisciplinary diagnostic process for determining the medical, psychological, and functional abilities of frail older adults to develop a coordinated, integrated plan for treatment and long-term follow-up. ${ }^{10}$

The CGA is associated with adverse health events such as death, disability, institutionalization, hospitalization, and falls $;^{10-13}$ therefore, the CGA is strongly associated with health status. The Yoitoko check-up assesses health improvements in older adults, such as the maintenance and improvement of items included in the CGA, and aims to promote positive behavioral changes related to these CGA items.

After completing a self-administered questionnaire and a simple measurement of function in the Yoitoko checkup, older adults received positive feedback concerning their current residual function, ie, good aspects of the assessment were highlighted. Providing encouragement has been reported to promote improved performance either directly or indirectly through increased motivation. ${ }^{14-16}$ Through applying these results, it is hoped that the Yoitoko check-up would motivate older adults to improve their health and encourage positive behavior change. The Yoitoko check-up was first applied in Yumesaki-cho, Himeji, Hyogo, Japan, in December 2018 in collaboration with local governments and universities. Since it is still in its early stages of development and implementation, its effectiveness must be evaluated hereafter. In case this novel style of health check-up is found to be effective, this new tool may support the creation of a more sustainable society through health promotion and preventive care. The Yoitoko check-up has the potential to contribute to the achievement of the SDGs.

In this first report on the Yoitoko check-up, we introduce the details of this check-up. To explore its value of continuous development and evaluation in the future, we evaluated the short-term changes in scores on the CGA items for the participants of this check-up.

\section{Materials and Methods \\ The Yoitoko Check-Up Composition of the Yoitoko Check-Up}

The Yoitoko check-up comprised a measurement of health status and positive feedback. Health status was measured using a self-administered questionnaire and a simple measurement of function. The questionnaire consisted of the following assessments: (i) participant characteristics, such as medical history, occupational history, and the number of family members living together; (ii) the Medical Outcomes Study 8-item Short-Form Health Survey [SF$8]$ to assess health-related quality of life (QOL), ${ }^{17,18}$ (iii) the Tokyo Metropolitan Institute of Gerontology Index of Competence [TMIG-IC] to assess high-level functional capacity, ${ }^{19}$ (iv) the Health-related Community Social Capital Scale [HrCSCS] to assess a participant's relationship with society ${ }^{20}$ and the Japanese version of the abbreviated Lubben Social Network Scale [LSNS-6]; ${ }^{21,22}$ (v) the Japanese version of the Geriatric Depression Scale-15 
[GDS-15] to assess mental health; ${ }^{23,24}$ (vi) the Subjective Index of Masticatory Ability ${ }^{25-27}$ and the 10-item Eating Assessment Tool [EAT-10] $]^{28,29}$ to assess oral health. The simple measurement of function involved the following: (i) a Timed Up \& Go test (TUG) to assess motor function $^{30}$ and (ii) the Oral Diadochokinesis [ODK] test $^{31}$ and a $30 \mathrm{~mL}$ water swallowing test [WST] ${ }^{32,33}$ to assess oral function. In addition to these measurements, brief-type self-administered diet history questionnaire ${ }^{34}$ for the assessment of daily diet, and the $5-\operatorname{Cog} \operatorname{test}^{35}$ for the assessment of cognitive function were provided as optional assessments since they contained many questions that it was not considered appropriate for all the participants to answer. Based on the results of the health status measurements, a participant received encouraging, affirmative feedback regarding positive findings.

\section{Details of Each Measurement Item Based on CGA}

The SF-8 is an index to evaluate general aspects of healthrelated QOL, ${ }^{17,18}$ comprising 8 dimension questions, namely, general health perception, physical functioning, role-physical (role limitations because of physical health), bodily pain, vitality, social functioning, mental health, and role-emotional (role limitations because of emotional problems). For each question, a score is calculated according to the prescribed scoring procedure. The physical component summary (PCS) and the mental component summary (MCS) scores are calculated, based on the scores of the 8 questions. The PCS and MCS scores were used in this study. The highest scores for each were 70.02 and 70.16 for the PCS and the MCS, respectively, with a high score indicating a high QOL.

The TMIG-IC index evaluates high-level functional capacity, ${ }^{19}$ and is comprised of 13 items, namely, 5 items on instrumental activity of daily living, 4 items on effectance, and 4 items on social role. Each question required a yes or no response, with a yes response scoring 1 point, and a total score of between 0 and 13 was calculated. A high total score indicated a high high-level functional capacity.

The HrCSCS is an index of social capital at the community level and was developed by the Japan Gerontological Evaluation Study Project. ${ }^{20}$ It is comprised of 3 elements, namely, civic participation, social cohesion, and reciprocity. Civic participation is assessed on a 6-point scale in relation to the frequency of participation in 5 groups (volunteer, sports, hobby, study or cultural, and skills teaching), with 1 point for participation at least once a month, for a total of 5 possible points. Social cohesion was assessed on a 5-point scale, ranging from strongly agree to strongly disagree on 3 items: community trust, norms of reciprocity, and community attachment. A total of 3 points are attributed for a strong or moderately agreeable score of 1 . Reciprocity is calculated through selecting the relationship (eg, spouse, child) that applies to the 3 items, namely, receiving emotional support, providing emotional support, and receiving instrumental support. A total of 3 points is given, with 1 point attributed even when only 1 person is involved in relation to each question. These civic participation, social cohesion, and reciprocity scores are added together to arrive at a total score ranging from 0 to 11 points. The higher the total score, the higher the social capital.

The LSNS-6 was developed to screen for social isolation. $^{21,22}$ The index comprises 3 questions to assess social ties, family, and friendships. A participant is asked questions concerning the number of people whom the participant has come into contact with at least once a month, the number of people the participant feels comfortable enough to talk with about private matters, and the number of people to whom the participant feels close enough to ask for help. Each question is answered on a 6-point scale ranging from 0 to 5 points, and a total score ranging from 0 to 30 points is calculated. A high total score suggests strong social ties.

The GDS-15 is a frequently used tool to screen for depression among older adults. ${ }^{23,24}$ It comprises 15 questions that are answered as either 'yes' or "no", with responses of "yes" in 10 items and responses of "no" in 5 items suggesting a depressive state. A total score of between 0 and 15 points is calculated, with 1 point for each choice. A low total score indicates a healthy mental status.

The SIMA includes 5 hard food groups (group 1, bananas, corned beef, and tofu; group 2, boiled rice and boiled asparagus; group 3, raisins and pickles; group 4, raw carrots, celery, and boiled ham, and; group 5, prepared shredded squid and yellow pickled radish). A total score of between 0 and 5 is calculated, with 1 point attributed each time a participant is able to bite and chew the selected food options. ${ }^{25-27}$ A high total score indicates a strong masticatory ability.

The EAT-10 is a tool used to screen for dysphagia. ${ }^{28,29}$ The questionnaire comprises 10 questions, each of which is answered on a 5-point scale ( 0 points, no difficulty; 4 points, severe difficulty). Each score is added and a total 
score of between 0 and 40 is calculated. A lower total score indicated that swallowing function has been retained.

The TUG is a balance test to assess functional mobility among older adults. ${ }^{30}$ The time taken between sitting comfortably on a chair with an armrest, walking 3 meters at a comfortable speed, turning back, and sitting down again completely is measured. In the original method, a participant is instructed to walk at a comfortable speed; however, another method involves a participant being instructed to walk as fast as possible. ${ }^{36}$ For the Yoitoko check-up, the original method was used to measure the time required (in seconds) to undertake the TUG, taking into account the risk of participants falling. A shorter time required indicates that functional mobility has been retained.

The ODK is an oral function test used to evaluate tongue-lip motor function. ${ }^{31} \mathrm{~A}$ participant is asked to repeat 1 of the syllables/pa/,/ta/or/ka/as quickly as possible in 5 seconds, and the number of syllable repetitions is measured. The syllable repetition rate per second is used for evaluation of oral function. In this study, the syllable repetition rate was measured using KENKOU-KUN (Takei Scientific Instruments Co., Ltd., Niigata, Japan). A fast speed indicates that tongue-lip motor function has been retained.

The $30-\mathrm{mL}$ WST is used to assess participants' swallowing function. ${ }^{32,33} \mathrm{~A}$ participant is instructed to swallow $30 \mathrm{~mL}$ of water (at room temperature) in the usual manner. Swallowing in 1 gulp without choking and within 5 seconds is considered normal. In this study, the presence or absence of any abnormal test findings was considered as a categorical variable.

\section{Positive Feedback to Participants}

Feedback to participants was conducted in 4 steps, as follows: Step 1. Praising participation, where the examiner admired the behavior in taking part in the Yoitoko checkup. Step 2. Praising individual findings. The examiner admired the high scoring items while confirming that all items had been fully completed. Scoring criteria for each item had been previously defined. An examiner asked a participant what they had specifically focused on in daily living regarding the items with a good score and, if anything was identified, the examiner acknowledged and supported the participant's efforts. The participant was encouraged to continue to maintain positive scores. Step 3. Praise for undertaking a conventional health check-up. While the Yoitoko check-up focused on the CGA, it did not replace a conventional health check-up. If a participant undertook conventional health check-ups, this action was positively reinforced. However, if a participant had not undergone a conventional health check-up, the examiner recommended they do so. Step 4. Summary. The examiner summarized the findings, re-emphasizing and praising the positive scores. The examiners were recommended to spend 10-15 min on the feedback, although actual feedback time was extended in some cases, depending on the examiner. If many items were scored highly, the examiner provided positive feedback on the items selected. On the other hand, if no item had a high score, the examiners asked the participants what they had specifically focused on in daily living and acknowledged the participant's efforts. Orientations related to the implementation of the Yoitoko check-up, including these feedback methods, were conducted in advance.

\section{Conducting the Yoitoko Check-Up}

This check-up was conducted as part of a community development project, in collaboration with local government and universities, and researchers and students from various academic fields (eg, economics) participated as well as medical professionals. In particular, students assisted the participants to complete the self-administered questionnaire, they measured participants' functions, and independently provided positive feedback. Given this check-up tool was developed with low cost, safety, and convenience in mind, it did not require invasive medical tests nor require an examiner to have a high level of medical knowledge. An examiner was able to provide feedback through undertaking basic pre-training. Therefore, we consider that the Yoitoko check-up is highly generalizable.

The Yoitoko check-up was started for older adults aged $\geq 65$ years in 2018. To date, the check-up has been conducted on 4 occasions (in December 2018, and in March, June, and September 2019) at Yamanouchi, YumesakiCho, Himeji city, Hyogo prefecture, Japan. Yamanouchi had about 600 citizens in $40.28 \mathrm{~km}^{2}$ and over $50 \%$ were $\geq 65$ years. Group activities for older adults living in this area are held monthly. Approximately $30-50$ older adults had participated in the group activities each time, and the Yoitoko check-ups were conducted for participants interested in undertaking the check-up on a particular day as part of these group activities. The first, second, and third check-ups were held at a regional community center, and the fourth check-up was undertaken at a nearby 
gymnasium, on a larger scale. The participants could take the Yoitoko check-up repeatedly, and some participants undertook more $>1$ check-up.

\section{Study Design}

This before-after study used participant data from the first, second, and subsequent Yoitoko check-ups to evaluate its feasibility. This report has been written with reference to the Strengthening the Reporting of Observational Studies in Epidemiology guidelines. ${ }^{37}$

\section{Study Participants}

This study comprised participants who had previously undertaken a Yoitoko check-up who met all the inclusion and exclusion criteria. The inclusion criteria comprised older adults aged $\geq 65$ years, and those who had undertaken a Yoitoko check-up on $\geq 2$ occasions. Exclusion criteria comprised those who declined to participate in this study.

\section{Measures}

We retrospectively analyzed the check-up data excluding the brief-type self-administered diet history questionnaire and the 5-Cog test, and the following data were used: demographics (age and sex), self-administered questionnaires involving medical history (presence of disease and smoking status), employment status, co-habitation status, and the SF8, TMIG-IC, HrCSCS, LSNS-6, GDS-15, SIMA, and EAT10 assessment tools, and; simple measures of functioning, namely, the TUG, the ODK, and the $30 \mathrm{~mL}$ WST. Presence of disease, smoking status, employment status, and cohabitation status were considered to be categorical variables.

\section{Study Size}

The population of this study comprised participants who had previously undertaken the Yoitoko check-up. Because we included 84 participants in the study size, we did not establish the number of participants based on statistical estimates.

\section{Statistical Method}

We first obtained descriptive statistics concerning participant background and outcome items. A paired $t$-test was then used to calculate the mean change from baseline for each variable and its $95 \%$ confidence interval (CI) by the number of visits (eg, 2nd visit vs baseline, or 3rd visit vs baseline). For the $30-\mathrm{mL}$ WST, we created a $2 \times 2$ table with the presence or absence of an abnormality as the objective variable, and the first visit and each visit as the explanatory variables. The odds ratio and its $95 \%$ CI were calculated using the McNemar test. A complete case analysis was performed to take missing data into account. All statistical analyses were performed using Stata MP version 15 (StataCorp LLC, College Station, TX, USA).

\section{Ethical Considerations}

This was an observational study using existing data; therefore, written consent was not sought from individual study participants. However, an opt-out format was adopted to ensure appropriate disclosure of information concerning the study and to provide an opportunity for study participants to decline enrollment. Participants were able to make inquiries or opt-out using the telephone, email, mail, or through contacting the research office. This study was conducted in accordance with the Declaration of Helsinki and was approved by the Institutional Review Board of Kobe University Graduate School of Medicine (number B200168). The datasets used and/or analyzed during the current study are available from the corresponding author on reasonable request.

\section{Results}

Between December 2018 and September 2019, a total of 4 Yoitoko check-ups were conducted, involving 84 individuals. Of these, data concerning 16 (19.0\%) participants who met the inclusion criteria were analyzed (Figure 1). The mean (standard deviation [SD]) age of the participants was 75.3 (4.7) years, and $6(37.5 \%)$ participants were male. Six (37.5\%) participants had undergone 3 checkups and no participants had undergone 4 check-ups (Table 1). The characteristics of these participants did not significantly differ from those of a single visitor of Yoitoko check-ups aged $\geq 65$ years. ( $\underline{\text { S1 Table }}$ ).

Table 2 shows a comparison of the first and second check-up results among those who had undergone $\geq 2$ Yoitoko check-ups. The mean TMIG-IC score increased by $0.9(95 \%$ CI, 0.2-1.5) points from baseline. Furthermore, the SF-8, HrCSCS, GDS-15, SIMA, EAT10 , and TUG scores altered favorably, although the differences were not statistically significant. Table 3 shows a comparison of the first and third check-up results among those who had undergone $\geq 3$ Yoitoko check-ups. No statistically significant differences were detected for any of the items. However, the TMIG-IC score (95\% CI) shifted in a favorable direction by $0.5(-0.9-1.9)$ points, showing a consistent trend. With respect to the $30-\mathrm{mL}$ WST, all participants were able to swallow without difficulty in the second and third visits, and the odds ratio could not be calculated. 


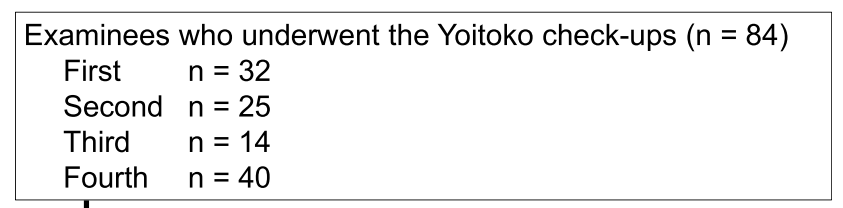

$\longrightarrow \begin{array}{ll}\text { Excluded } & \\ \text { Age }<65 \text { years } & \mathrm{n}=68 \\ \text { One-time visit } & \mathrm{n}=46 \\ \text { Request for non-participation } & \mathrm{n}=0\end{array}$

Study participants $\quad \mathrm{n}=16$

Figure I Flow chart with details of study participation.

\section{Discussion}

A conventional health check-up has shortcomings that it does not always improve the participants' motivation for health promotion. Therefore, we developed Yoitoko check-

Table I Characteristics of Study Participants Who Had Undergone $\geq 2$ Yoitoko Check-Ups in Hyogo, 2018-2019 $(n=16)$

\begin{tabular}{|l|c|}
\hline & $\mathbf{n}(\%)$ \\
\hline Age- mean (SD) & $75.3(4.7)$ \\
\hline Sex: male & $6(37.5)$ \\
\hline Present diseases & $14(87.5)$ \\
Hypertension & $7(43.8)$ \\
Hyperlipidemia & $4(25.0)$ \\
Hyperuricemia & $0(0.0)$ \\
Diabetes mellitus & $1(6.3)$ \\
Cerebrovascular disease & $2(12.5)$ \\
Heart disease & $4(25.0)$ \\
Kidney disease & $2(12.5)$ \\
Liver disease & $0(0.0)$ \\
Depression & $2(12.5)$ \\
\hline Number of diseases & \\
One & $6(37.5)$ \\
Two & $5(31.3)$ \\
$\geq$ three & $1(6.3)$ \\
\hline Smoking status & $14(87.5)$ \\
Never smoked & $10(62.5)$ \\
Former smoker & $6(37.5)$ \\
Current smoker & $0(0.0)$ \\
\hline Currently employed & $1(6.3)$ \\
\hline Co-habitation & $0(0.0)$ \\
\hline Number of check-ups & \\
3 & \\
4 & \\
\hline
\end{tabular}

Abbreviation: SD, standard deviation.
Table 2 Comparison of the First and Second Results Among Participants Who Had Undergone $\geq 2$ Yoitoko Check-Ups $(n=16)$

\begin{tabular}{|c|c|c|c|c|}
\hline & \multirow[b]{2}{*}{$\mathbf{n}$} & $\begin{array}{l}\text { First } \\
\text { Visit }\end{array}$ & $\begin{array}{l}\text { Second } \\
\text { Visit }\end{array}$ & \multirow[b]{2}{*}{$\begin{array}{l}\text { Difference } \\
(95 \% \mathrm{Cl})\end{array}$} \\
\hline & & Mean (SD) & Mean (SD) & \\
\hline \multicolumn{5}{|l|}{ SF-8 } \\
\hline PCS & 14 & $42.8(4.7)$ & $44.0(6.9)$ & I.2 (-3.0-5.3) \\
\hline MCS & 14 & $51.8(5.4)$ & $52.7(4.1)$ & $0.9(-2.2-4.0)$ \\
\hline TMIG-IC & 15 & II.3 (I.8) & $12.2(\mathrm{I} . \mathrm{I})$ & $0.9(0.2-1.5)$ \\
\hline $\mathrm{HrCSCS}$ & 12 & $7.2(1.4)$ & $7.4(1.2)$ & $0.3(-0.4-0.9)$ \\
\hline LSNS-6 & 16 & $16.8(5.1)$ & $16.5(4.6)$ & $-0.3(-2.2-1.7)$ \\
\hline GDS-I5 & 14 & $4.2(3.2)$ & $3.7(2.9)$ & $-0.5(-2.3-1.3)$ \\
\hline SIMA & 16 & $4.6(0.7)$ & $4.7(0.7)$ & $0.1(-0.1-0.4)$ \\
\hline EAT- 10 & 15 & $1.9(3.4)$ & I.4 (I.8) & $-0.5(-2.1-1.1)$ \\
\hline TUG & 16 & 9.4 (1.9) & $8.9(2.3)$ & $-0.5(-1.4-0.5)$ \\
\hline \multicolumn{5}{|l|}{ ODK } \\
\hline $\mathrm{pa}$ & 16 & $5.5(1.2)$ & $5.1(1.6)$ & $-0.5(-1.3-0.4)$ \\
\hline ta & 16 & $5.0(1.3)$ & $4.6(1.7)$ & $-0.4(-1.4-0.7)$ \\
\hline \multirow[t]{3}{*}{ ka } & 16 & $4.8(1.4)$ & $4.7(1.6)$ & $-0.1(-0.8-0.5)$ \\
\hline & & $\begin{array}{l}\text { First } \\
\text { visit }\end{array}$ & $\begin{array}{l}\text { Second } \\
\text { visit }\end{array}$ & \multirow[b]{2}{*}{ OR $(95 \% \mathrm{Cl})$} \\
\hline & & n (\%) & n (\%) & \\
\hline $\begin{array}{l}\text { 30-mL WST: } \\
\text { normal }\end{array}$ & 16 & $14(87.5)$ & $16(100.0)$ & ND \\
\hline
\end{tabular}

Abbreviations: $\mathrm{Cl}$, confidence interval; EAT-10, 10-item Eating Assessment Tool, GDS-15, Geriatric Depression Scale-15; HrCSCS, Health-related Community Social Capital Scale; LSNS-6, the abbreviated Lubben Social Network Scale; MCS, Mental Component Summary; ND, not detectable; ODK, Oral Diadochokinesis; OR, odds ratio; PCS, physical component summary; SD, standard deviation; SF-8, the Medical Outcomes Study 8-item Short-Form Health Survey; SIMA, the Subjective Index of Masticatory Ability; TMIG-IC, Tokyo Metropolitan Institute of Gerontology Index of Competence; TUG, Timed Up \& Go Test; WST, Water Swallowing Test.

up providing positive feedback to participants based on a CGM assessment, and described the details in this report. We subsequently compared the before-after findings regarding the specific check-up items and assessed the short-term behavioral changes in comprehensive functioning resulting from applying the Yoitoko check-up as an initial stage evaluation for its potential for future development. The results showed an increase in TMIG-IC scores that measure high-level functional capacity between first and second Yoitoko check-ups. The increase in the TMIGIC scores was minimal; however, the increase was significant. Fujiwara et al re-tested the TMIG-IC after 1 month 
Table 3 A Comparison of the First and Third Check-Up Results Among Those Who Had Undergone 3 Yoitoko Check-Ups $(n=6)$

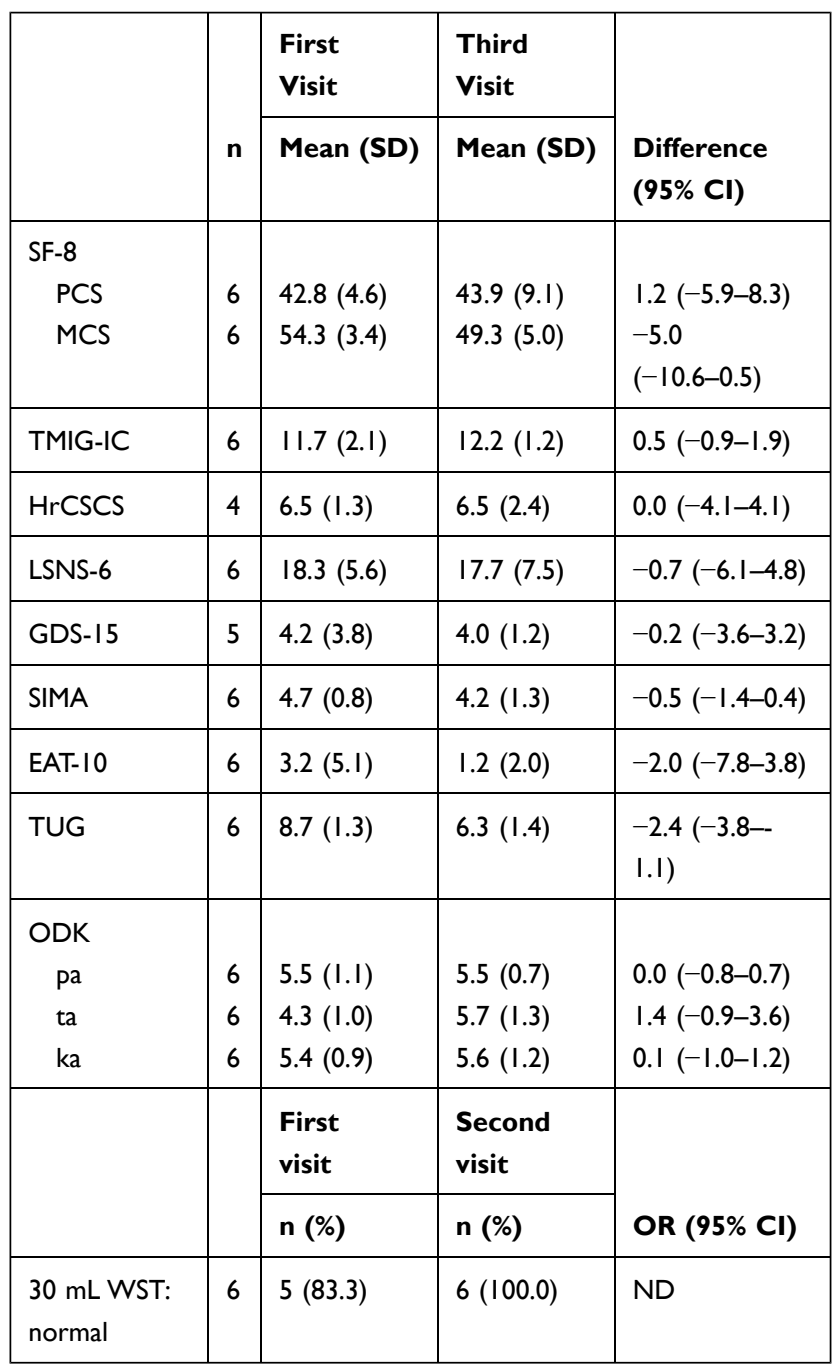

Abbreviations: $\mathrm{Cl}$, confidence interval; EAT-10, 10-item Eating Assessment Tool, GDS-15, Geriatric Depression Scale-15; HrCSCS, Health-related Community Social Capital Scale; LSNS-6, the abbreviated Lubben Social Network Scale; MCS, Mental Component Summary; ND, not detectable; ODK, Oral Diadochokinesis; OR, odds ratio; PCS, physical component summary; SD, standard deviation; SF-8, the Medica Outcomes Study 8-item Short-Form Health Survey; SIMA, the Subjective Index of Masticatory Ability; TMIG-IC, Tokyo Metropolitan Institute of Gerontology Index of Competence; TUG, Timed Up \& Go Test; WST, Water Swallowing Test.

and the mean score was 12.54 for both the first and second time check-ups, ie there was no change in the mean score. ${ }^{38}$ Moreover, there are other studies evaluating score changes longitudinally, which had evaluated the presence or absence of a score decrease. ${ }^{39-43}$ These results differed given that the mean score increased in our study. In addition, although no statistically significant differences were found, there were desirable shifts in scores in relation to several items. These results suggest that the Yoitoko check-up would be worthy of long-term evaluation in the future.
The changes towards desirable behavior observed in this study have also been reported in other studies, for example, in terms of providing education and praise, and in showing that performance measures improved either directly or indirectly when promoting increased motivation. ${ }^{14-16}$ Bandura stated that an expectation that taking action would lead to positive outcomes for oneself (outcome expectations) and confidence in being able to perform an action well (efficacy expectations) were important in enhancing behavioral change. Bandura also identified 4 sources of enhancing efficacy expectations: performance accomplishments, vicarious experience, verbal persuasion, and emotional arousal. ${ }^{44}$ Praise and positive feedback appeared to enhance efficacy expectations via verbal persuasion and emotional arousal. In the same way, through praising a desired behavior during the Yoitoko check-up, the importance of the current lifestyle was emphasized, and older adults may have been encouraged to make even more beneficial lifestyle changes. Although these mechanisms are speculative, the findings in this study suggested that, through praising desirable behavior, it was possible to influence behavioral changes among older adults. Well-evaluated behavioral approaches for health promotion include the health action process approach $^{45}$ and motivational interviewing. ${ }^{46}$ However, they require specialized knowledge and skills to apply them properly. On the other hand, Yoitoko check-up focuses on providing positive feedback on a good lifestyle. It may be more easily applied than previous behavioral approaches. In fact, university students with little expertise provided feedback in the Yoitoko check-up. If the effectiveness of Yoitoko check-up is established, it may be possible to implement a step-by-step approach in which Yoitoko check-up is administered to a broad population and the behavioral approaches are implemented in a highrisk population.

Our study results only showed a significant improvement in the TMIG-IC score, and no statistically significant improvement was identified in terms of the other items. The TMIG-IC is a measure of life function and spontaneous changes can occur relatively easily, with other items less likely to improve in the short term. The SF-8 assesses physical and mental summary scores, which include items such as physical function and pain, where short-term improvements may be more difficult to achieve. With regard to social connections as assessed using the HrCSCS and the LSNS-6, increasing the number of a participant's close contacts would be difficult in the 
short term, even if the participant was able to voluntarily increase their involvement in social activities. With regard to the GDS-15, control of depressive emotions is likely to be difficult in the short term. It is challenging to obtain improvement in physical functions such as masticatory ability, swallowing function, vocal ability, and walking function within a short timeframe without appropriate training, guidance, and increased physical effort. In this study, we found no significant improvement in many of the scores; however, we did observe a generally positive shift in scores. Therefore, it is possible that these scores may improve further with longer-term observation. The CGA, based on health screening items, suggests possible health indicators among older adults ${ }^{10-13}$ such that increasing the CGA score may lead to improvements in health. Future research is needed; however, the findings in this study suggest that the positive feedback in terms of promoting desirable behavior through applying the Yoitoko check-up could foster health improvements among older adult participants.

This study has several limitations that need to be resolved in future research. First, the number of participants in the study was small, thus limiting its power. However, assuming that the level of alpha was 0.05 and power was 0.8 , the number of participants required to detect the difference in TMIG-IC score, which is the main finding, was estimated to be 14. Therefore, the number of participants in this study would have been sufficient to detect the difference in TMIGIC scores. Second, this study could contain a selection bias. The study participants were only $19.0 \%$ of the target population, and we could have focused more on the healthconscious people. The absence of current smokers among the study participants suggests a health-conscious bias. ${ }^{47}$ Therefore, generalization from this study should be considered carefully. However, the characteristics of single and multiple time participants did not significantly differ, thus the effect of selection bias may be small. We assumed that participants would take the Yoitoko check-up once a year as they do for conventional checkups; however, the fact that $19.0 \%$ of the participants took it again at intervals of 3 to 6 months may indicate that there are high participants' expectations for the Yoitoko check-ups. Third, there was no comparison group, which was based on the characteristics of the study design; In this study, it was not possible to assess changes in TMIG-IC score without intervention within the same community. Therefore, we could not discern whether this result was caused by the intervention, spontaneously without the intervention, or by other factors such as confounding factors and effect modification. However, the TMIG-IC score usually remains unchanged or decreases over time ${ }^{38-43}$ which differs from the score change in our study. Comparison with these existing data could support that the result was not caused spontaneously without intervention. Besides these significant limitations, it is possible that social desirability bias may have occurred due to some subjective measurement items. ${ }^{48}$ However, since the measured items were indicators whose validity and reliability have been verified in previous studies, we consider that eliminating the measurement bias is should have been addressed. This study could be categorized into a feasibility study. The influence of positive feedback in Yoitoko-checkups has been evaluated by analyzing the difference in the measurements between the first and second visits. Therefore, in the future, it will be necessary to examine its effect on the robust behavioral change model from the previous study. This short-term and exploratory evaluation has suggested important factors that support the continuation of the novel Yoitoko check-up. Implementation of the Yoitoko check-up has only just been initiated, and it is necessary to address the issues as part of the ongoing evaluation. Hence, it will be necessary to increase the number of participants in the study, prepare a control group, and perform detailed analysis with adjusted confounding and effect modification in the future.

\section{Conclusion}

We developed the Yoitoko check-up and introduced the details of it. Then, initial evaluation of the novel style of health checkup showed that, even in the short term, through providing positive feedback on the good points of the check-up, the Yoitoko check-up could improve high-level functional capacity and increase the motivation of older adults to improve their health and promote positive behavioral changes. Applying the Yoitoko check-up might contribute to the achievement of SDGs through promoting lifelong health and preventive care and provide a new tool to help build a sustainable society. The Yoitoko check-up has only recently been developed and its benefits have yet to be fully assessed. The results of this study provide evidence to support its continued implementation and further validation. It is hoped that its usefulness will be evaluated from various perspectives in the future.

\section{Abbreviations}

SDG, Sustainable Development Goals; CGA, Comprehensive Geriatric Assessment; TMIG-IC, Tokyo Metropolitan Institute of Gerontology Index of 
Competence; HrCSCS, Health-related Community Social Capital Scale; LSNS, Lubben Social Network Scale; GDS15, Geriatric Depression Scale-15; EAT, Eating Assessment Tool; TUG, Timed Up \& Go test; ODK, Oral Diadochokinesis; WST, Water Swallow Test; PCS, Physical component summary; MCS, Mental component summary.

\section{Acknowledgments}

We wish to thank all the community development project members (Tomoko Kinugasa, Kobe University; Makoto Usami, Konan Women's University; Isamu Saeki, Konan Women's University; Nobuko Amano, Konan Women's University; Toshiyuki Kohri, Konan Women's University; Sana Inoue, Ritsumeikan University, and; Nozomi Okamoto, Hyogo University of Teacher Education) for their cooperation in conducting the Yoitoko check-ups. We also would like to thank Editage (www.editage.com) for their English language editing services.

\section{Funding}

This research was funded by the Nippon Life Insurance Foundation's 2018 Research Grant for Practical Issues in Aging Society, grant number 18.

\section{Disclosure}

The authors report no conflicts of interest in this work.

\section{References}

1. United Nations, Department of Economic and Social Affairs, Population Division. World Population Prospects 2019: highlights (ST/ESA/SER.A/423); 2019. Available from: https://population.un. org/wpp/Publications/Files/WPP2019_Highlights.pdf. Accessed Feb 16, 2021.

2. United Nations, Department of Economic and Social Affairs, Population Division. World Population Ageing 2019: highlights (ST/ ESA/SER.A/430); 2020. Available from: https://www.un.org/en/devel opment/desa/population/publications/pdf/ageing/ WorldPopulationAgeing2019-Report.pdf. Accessed Feb 16, 2021.

3. Marengoni A, Angleman S, Melis R, et al. Aging with multimorbidity: a systematic review of the literature. Ageing Res Rev. 2011;10 (4):430-439. doi:10.1016/j.arr.2011.03.003

4. Minister of Health, Labour and Welfare. A Basic Direction for Comprehensive Implementation of National Health Promotion; 2012. Available from: https://www.mhlw.go.jp/file/06-Seisakujouhou -10900000-Kenkoukyoku/0000047330.pdf. Accessed Feb 16, 2021.

5. Kohro T, Furui Y, Mitsutake N, et al. The Japanese national health screening and intervention program aimed at preventing worsening of the metabolic syndrome. Int Heart J. 2008;49(2):193-203. doi:10.1536/ihj.49.193

6. Nakao YM, Miyamoto Y, Ueshima K, et al. Effectiveness of nationwide screening and lifestyle intervention for abdominal obesity and cardiometabolic risks in Japan: the metabolic syndrome and comprehensive lifestyle intervention study on nationwide database in Japan (MetS ACTION-J study). PLoS One. 2018;13(1):e190862. doi:10.1371/journal.pone. 0190862
7. Tsushita K, Hosler AS, Miura K, et al. Rationale and descriptive analysis of specific health guidance: the nationwide lifestyle intervention program targeting metabolic syndrome in Japan. J Atheroscler Thromb. 2018;25(4):308-322. doi:10.5551/jat.42010

8. Minister of Health, Labour and Welfare. [Towards a national rollout of health services based on the characteristics of the elderly (in Japanese)]; 2018. Available from: https://www.mhlw.go.jp/content/ 12401000/000422882.pdf. Accessed Feb 16, 2021. Japanese

9. Minister of Health, Labour and Welfare. Summary Report of Comprehensive Survey of Living Conditions 2016; 2017. Available from: https://www.mhlw.go.jp/english/database/db-hss/dl/report_gai kyo_2016.pdf. Accessed Feb 16, 2021.

10. Ellis G, Whitehead MA, O'Neill D, Langhorne P, Robinson D. Comprehensive geriatric assessment for older adults admitted to hospital. Cochrane Database Syst Rev. 2011;7. doi:10.1002/ 14651858.CD006211.pub2

11. Parker SG, McCue P, Phelps K, et al. What is Comprehensive Geriatric Assessment (CGA)? An umbrella review. Age Ageing. 2018;47(1):149-155. doi:10.1093/ageing/afx166

12. Chiu CJ, Cheng YY. Utility of Geriatric Syndrome Indicators for Predicting Subsequent Health Care Utilization in Older Adults in Taiwan. Int $J$ Environ Res Public Health. 2019;16(3):456. doi:10.3390/ijerph16030456

13. Mishra AK, Skubic M, Popescu M, et al. Tracking personalized functional health in older adults using geriatric assessments. BMC Med Inform Decis Mak. 2020;20(1):270. doi:10.1186/s12911-020-01283-y

14. Catano VM. Relation of Improved Performance through Verbal Praise to Source of Praise. Percept Mot Skills. 1975;41(1):71-74. doi:10.2466/pms.1975.41.1.71

15. Henderlong J, Lepper MR. The effects of praise on children's intrinsic motivation: a review and synthesis. Psychol Bull. 2002;128 (5):774-795. doi:10.1037/0033-2909.128.5.774

16. Sugawara SK, Tanaka S, Okazaki S, Watanabe K, Sadato N. Social Rewards Enhance Offline Improvements in Motor Skill. PLoS One. 2012;7(11):e48174. doi:10.1371/journal.pone.0048174

17. Fukuhara S, Suzukamo Y. Manual of the SF-8 Japanese Version, (In Japanese). Kyoto: Institute for Health Outcomes and Process Evaluation Research; 2004.

18. Ware JE. How to Score and Interpret Single-Item Health Status Measures: A Manual for Users of the of the SF-8 Health Survey: (With a Supplement on the SF-6 Health Survey). Boston, MA: Lincoln, RI: QualityMetric, Inc.; 2001.

19. Koyano W, Shibata H, Nakazato K, Haga H, Suyama Y. Measurement of competence: reliability and validity of the TMIG Index of Competence. Arch Gerontol Geriatr. 1991;13(2):103-116. doi:10.1016/0167-4943(91)90053-S

20. Saito M, Kondo N, Aida J, et al. Development of an instrument for community-level health related social capital among Japanese older people: the JAGES Project. J Epidemiol. 2017;27(5):221-227. doi:10.1016/j.je.2016.06.005

21. Kurimoto A, Awata S, Ohkubo T, et al. [Reliability and validity of the Japanese version of the abbreviated Lubben Social Network Scale (in Japanese)]. Nippon Ronen Igakkai Zasshi. 2011;48(2):149-157. doi:10.3143/geriatrics.48.149

22. Lubben J, Blozik E, Gillmann G, et al. Performance of an abbreviated version of the Lubben Social Network Scale among three European community-dwelling older adult populations. Gerontologist. 2006;46 (4):503-513. doi:10.1093/geront/46.4.503

23. Sugishita K, Sugishita M, Hemmi I, Asada T, Tanigawa T, Validity A. Reliability Study of the Japanese Version of the Geriatric Depression Scale 15 (GDS-15-J). Clin Gerontol. 2017;40(4):233-240. doi:10.1080/07317115.2016.1199452

24. Burke WJ, Roccaforte WH, Wengel SP. The short form of the Geriatric Depression Scale: a comparison with the 30-item form. J Geriatr Psychiatry Neurol. 1991;4(3):173-178. doi:10.1177/ 089198879100400310 
25. Yanagisawa Y, Tamura A, Teramoto Y, Akasaka M. [A classification of foods by the amount of masticatory action involved]. Shoni Shikagaku Zasshi. 1989;27(1):74-84. Japanese.

26. Hirano H, Ishiyama N, Watanabe I, Nasu I. Masticatory ability in relation to oral status and general health on aging. $J$ Nutr Health Aging. 1999;3(1):48-52.

27. Okamoto N, Amano N, Nakamura T, Yanagi M. Relationship between tooth loss, low masticatory ability, and nutritional indices in the elderly: a cross-sectional study. BMC Oral Health. 2019;19 (1):110. doi:10.1186/s12903-019-0778-5

28. Belafsky PC, Mouadeb DA, Rees CJ, et al. Validity and reliability of the Eating Assessment Tool (EAT-10). Ann Otol Rhinol Laryngol. 2008;117(12):919-924. doi:10.1177/000348940811701210

29. Wakabayashi H, Kayashita J. [Translation, reliability, and validity of the Japanese version of the 10-item Eating Assessment Tool (EAT-10) for the screening of dysphagia]. Jomyaku Keicho Eiyo. 2014;29(3):871-876.Japanese.

30. Podsiadlo D, The RS. timed "Up \& Go": a test of basic functional mobility for frail elderly persons. J Am Geriatr Soc. 1991;39 (2):142-148. doi:10.1111/j.1532-5415.1991.tb01616.x

31. Portnoy RA, Aronson AE. Diadochokinetic syllable rate and regularity in normal and in spastic and ataxic dysarthric subjects. $J$ Speech Hear Disord. 1982;47(3):324-328. doi:10.1044/jshd.4703.324

32. Kubota T, Mishima H, Hanada M, Namba I, Kojima Y. [Paralytic dysphagia in cerebrovascular disorder-screening tests and their clinical application]. Sogo Rehabilitation. 1982;10(2):271-276. Japanese.

33. Horiguchi S, Suzuki Y. Screening tests in evaluating swallowing function. JMAJ. 2011;54(1):31-34.

34. Kobayashi S, Murakami K, Sasaki S, et al. Comparison of relative validity of food group intakes estimated by comprehensive and brief-type self-administered diet history questionnaires against 16 d dietary records in Japanese adults. Public Health Nutr. 2011;14 (7):1200-1211. doi:10.1017/S1368980011000504

35. Sasaki M, Kodama C, Hidaka S, et al. Prevalence of four subtypes of mild cognitive impairment and APOE in a Japanese community. Int J Geriatr Psychiatry. 2009;24(10):1119-1126. doi:10.1002/gps.2234

36. Shumway-Cook A, Brauer S, Woollacott M. Predicting the Probability for Falls in Community-Dwelling Older Adults Using the Timed Up \& Go Test. Phys Ther. 2000;80(9):896-903. doi:10.1093/ptj/80.9.896

37. Elm E, Altman DG, Egger M, Pocock SJ, Gøtzsche PC, Vandenbroucke JP. Strengthening the reporting of observational studies in epidemiology (STROBE) statement: guidelines for reporting observational studies. BMJ. 2007;335(7624):806-808. doi:10.1136/ bmj.39335.541782.AD
38. Fujiwara Y, Shinkai S, Amano H, et al. [Test-retest variation in the Tokyo Metropolitan Institute of Gerontology Index of Competence in community-dwelling older people independent in daily living toward individual assessment of functional capacity]. Nihon Koshu Eisei Zasshi. 2003;50(4):360-367. Japanese.

39. Imamura H, Uchiyama E, Akiyama M, Kaneko I, Takebayashi T, Nishiwaki Y. Relationship of living arrangement with the decline in functional capacity in elderly people by gender: a longitudinal observational study. Environ Health Prev Med. 2020;25(1):15. doi:10.1186/s12199-020-00853-w

40. Sugai K, Michikawa T, Takebayashi T, Nishiwaki Y. Knee pain and future decline in higher-level functional competence in community-dwelling older Japanese: the Kurabuchi cohort study. Age Ageing. 2020;49(4):592-598. doi:10.1093/ageing/afaa024

41. Sato K, Kondo N, Kondo K. Rotating savings and credit association, its members' diversity, and higher-level functional capacity: a 3-year prospective study from the Japan Gerontological Evaluation Study. Geriatr Gerontol Int. 2019;19(12):1268-1274. doi:10.1111/ggi.13798

42. Cho S, Park S, Takahashi S, Yoshiuchi K, Shephard RJ, Aoyagi Y. Changes in and Interactions between Physical and Mental Health in Older Japanese: the Nakanojo Study. Gerontology. 2019;65 (4):340-352. doi:10.1159/000494383

43. Tomioka K, Kurumatani N, Saeki K. The differential effects of type and frequency of social participation on IADL declines of older people. PLoS One. 2018;13(11):e0207426. doi:10.1371/journal. pone. 0207426

44. Self-efficacy: BA. Toward a unifying theory of behavioral change. Psychol Rev. 1977;84(2):191-215. doi:10.1037/0033-295X.84.2.191

45. Schwarzer R, Hamilton K. Changing behavior using the health action process approach. In: Hagger MS, Cameron LB, Hamilton K, Hankonen N, Lintunen T, editors. The Handbook of Behavior Change. Cambridge: Cambridge University Press; 2020:89-103.

46. Rollnick S, Butler CC, Kinnersley P, Gregory J, Mash B. Motivational interviewing. BMJ. 2010;340:c1900. doi:10.1136/bmj. c1900

47. Shrank WH, Patrick AR, Brookhart MA. Healthy user and related biases in observational studies of preventive interventions: a primer for physicians. J Gen Intern Med. 2011;26(5):546-550. doi:10.1007/ s11606-010-1609-1

48. Nederhof AJ. Methods of coping with social desirability bias: a review. Eur J Soc Psychol. 1985;15(3):263-280. doi:10.1002/ ejsp. 2420150303
International Journal of General Medicine

\section{Publish your work in this journal}

The International Journal of General Medicine is an international, peer-reviewed open-access journal that focuses on general and internal medicine, pathogenesis, epidemiology, diagnosis, monitoring and treatment protocols. The journal is characterized by the rapid reporting of reviews, original research and clinical studies across all disease areas. The manuscript management system is completely online and includes a very quick and fair peer-review system, which is all easy to use. Visit http://www.dovepress.com/ testimonials.php to read real quotes from published authors. 